\title{
Development of chitosan-catechol conjugates as mucoadhesive polymer: assessment of acute oral toxicity in mice
}

\author{
Loveleen Kaur ${ }^{1}$ (D), Ritu Raj ${ }^{1}$, Ajay Kumar Thakur ${ }^{1(D)}$ and Inderbir Singh ${ }^{1, *(D)}$ \\ ${ }^{1}$ Chitkara College of Pharmacy, Chitkara University, Punjab, India. \\ *Correspondence: inderbir.singh@chitkara.edu.in
}

Received: June 6, 2020 Accepted: August 6, 2020

\begin{abstract}
Development of modified polymers is the focused area of research for developing stable, effective, sustainable and economical polymeric materials for developing different drug delivery systems. Modification of chitosan by catechol functionalization is useful for developing chitosan derivative with the improved mucoadhesive property. Present study was designed to perform single dose acute oral toxicity on chitosan-catechol conjugates in Swiss albino mice as per international guidelines. Oral administration of modified chitosan did not exhibit any significant change in body weight, behavioural pattern, haematology, food intake and clinical symptoms in the experimental animals. In the histopathological study, no pathological changes were observed in the vital organs of mice when administered perorally with $300 \mathrm{mg} / \mathrm{kg}$ and 2,000 mg/kg body-weight doses of chitosan-catechol polymeric conjugates. Overall, it was concluded from the acute oral toxicity study that the oral administration of chitosan-catechol conjugates in mice did not produce any toxicity. Hence, chitosan-catechol conjugates could be designated and recommended as safe polymeric material for developing different drug delivery systems.
\end{abstract}

Keywords: chitosan-catechol conjugate, acute oral toxicity, mice.

\section{Introduction}

Polymers are multifunctional excipients/materials playing a vital role in the development of different drug delivery systems. New polymers, processed polymers and modified polymers must comply with the regulatory requirements for safe and effective use in human beings [1]. International Pharmaceutical Excipients Council (IPEC) has published safety evaluation guidance for pharmaceutical excipients. IPEC and United States Food and Drug Administration (USFDA) have recommended preclinical testing strategies for short-term, mid-term and long-term clinical use of new excipients [2,3].

Chitosan is a natural polysaccharide comprising of D-glucosamine and N-acetyl-D-glucosamine units linked through 1,4- $\beta$-glycosidic bonds. It can be obtained by partial deacetylation of chitin from crustacean shells. Chitosan is available with different molecular weights (50-2,000 kDa), viscosities and degree of deacetylation (40-98\%) [4]. It is a mucoadhesive, nontoxic, biodegradable, biocompatible biopolymer having diverse applications in pharmaceutical, biotechnology, textile, agriculture, food and cosmetics industries [5,6]. Chitosan is potentially safe for developing oral drug delivery systems. Oral levels up to $6.75 \mathrm{~g}$ were reported to be safe for human consumption [7]. Kim et al. evaluated subacute toxicity of chitosan oligosaccharide in rats and reported no adverse effects at 2,000 $\mathrm{mg} / \mathrm{kg}$ dose [8]. Chitosan and carboxymethyl chitosan were reported to be safe and effective for developing carriers for oral delivery of insulin [9,10]. Chitosan itself possesses mucoadhesive property and numerous kinds of literature are available on attempts to further enhance its mucoadhesive property [11]. Catechol conjugation of chitosan is a promising method for enhancing its solubility, permeability and mucoadhesive property [12].

Present investigation was designed to perform acute oral toxicity (single dose) study of chitosan-catechol conjugates in mice. Various haematological, liver function, kidney function tests, physiological and feeding behaviour parameters were assessed during the study. Pathohistological analysis was performed for visualizing the cellular structural stability of different tissues following the oral administration of polymeric material.

\section{Materials and Methods Materials}


Low molecular weight chitosan (Molecular weigh 50,000-190,000 Da), 3, 4-Dihydoxyhydrocinnamic acid (98\%, HCA) and N-(3-Dimethylaminopropyl)-N'-ethylcarbodiimide hydrochloride (EDC) were procured from Sigma-Aldrich (St. Louis, MO, USA). All reagents and chemicals employed in the study were analytical grade and were used as received.

\section{Animals}

Swiss albino mice ( $26 \pm 2 \mathrm{~g}$ ) of either sex were procured from LLRUVAS, Hisar, India and were kept under standard housing conditions following balanced diet and water ad libitum. The animal study protocol was approved (IAEC/CCP/19/PR-017) by the animal ethics committee of the institute and following the guidelines of committee for the purpose of control and supervision of experiments on animals (CPCSEA), Government of India (Reg.No. 1181/PO/ReBi/S/08/CPCSEA).

\section{Synthesis and Characterization of Chitosan-Catechol Conjugates}

Chitosan-catechol conjugates were synthesized in our laboratory as per the directions and conditions presented in Figure 1. Chitosan $(3.25 \mathrm{mmol})$ was hydrated in $1 \mathrm{~N} \mathrm{HCl}$ followed by addition of sufficient amount of distilled water. The $\mathrm{pH}$ of the reaction mixture was adjusted with $\mathrm{NaOH}(5 \mathrm{~N})$ followed by the addition of HCA $(6.49$ mmol) with continuous stirring. EDC (6.49 mmol) dissolved in solution of ethanol and distilled water (1:1, v/v) was added dropwise. The reaction mixture was then dialysed in acidified distilled water $(\mathrm{pH}, \mathrm{HCl})$ for two days. The final product (chitosan-catechol conjugate) was freeze-dried and stored in a desiccator. Chitosan-catechol conjugates were represented as modified polymer having significant mucoadhesive properties rendering them to be useful for developing mucoadhesive drug delivery systems [13]. The surface morphology of chitosan and chitosan-catechol conjugates was investigated using a scanning electron microscope (SEM; JSM-6510LV, JEOL, Peabody, MA, USA). Spectrum of chitosan, catechol and chitosan-catechol conjugates was recorded by Fourier-transform infrared (FT-IR) spectrophotometer Alpha II model (Bruker, Billerica, MA, USA) using $\mathrm{KBr}$ disc method in the range of $3500-500 \mathrm{~cm}^{-1}$. X-ray power diffraction (XRD) analysis of samples was carried out employing X-ray diffractometer.

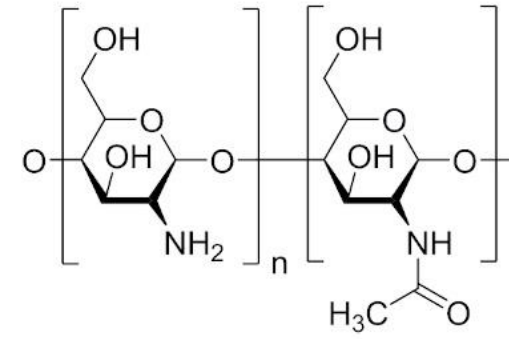

Chitosan<smiles>O=C(O)CCc1ccc(O)c(O)c1</smiles>

Hydrocaffeic acid 3,4-dihydroxy hydrocinnamic acid

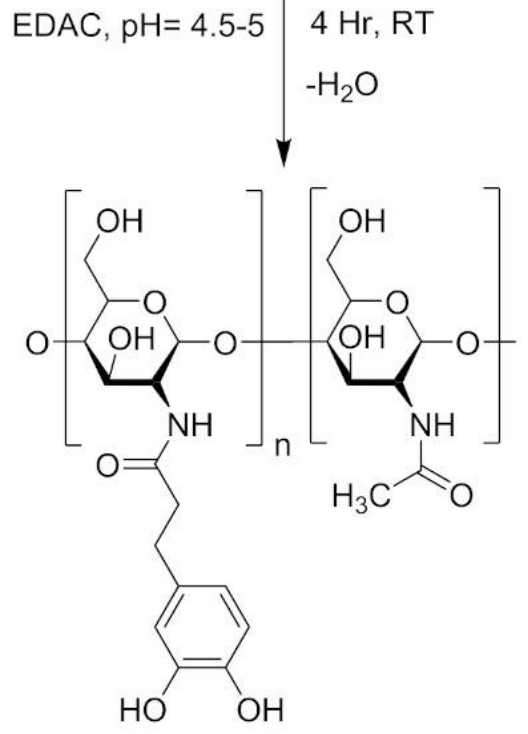

Chitosan-Catechol

Figure 1. Scheme for the synthesis of chitosan-catechol conjugates. 


\section{Experimental Procedure}

The single dose in vivo acute oral toxicity study on chitosan-catechol conjugates was performed as per Organization for Economic Co-operation and Development (OECD) 423 guidelines, with14-day study design and $300 \mathrm{mg} / \mathrm{kg}$ body-weight dose in Swiss albino mice. The detailed experimental schedule for the acute toxicity study is explained in Table 1 .

Table 1. In vivo acute oral toxicity schedule.

\begin{tabular}{|c|c|}
\hline S. No. & Experiment treatment schedule \\
\hline 1. & Group I Control $(0.9 \%$, w $/ \mathrm{v}$ saline, p.o.; $n=6)$ \\
\hline 2. & Group II (Chitosan-catechol conjugate $300 \mathrm{mg} / \mathrm{kg}$, p.o.; $n=3)$ \\
\hline 3. & Group III (Chitosan-catechol conjugate $2,000 \mathrm{mg} / \mathrm{kg}$, p.o.; $n=3)$ \\
\hline
\end{tabular}

Different single doses (300, 2,000 mg/kg body-weight) of chitosan-catechol conjugates were suspended in distilled water and were administered perorally to the mice using feeding needles made of stainless steel. Animals were observed individually for any signs of toxicity and behavioural changes for 4 hours after the dose administration followed by once daily monitoring for 14 days. Animals were checked daily for mortality and/or morbidity. Body weight of animals was recorded at the time of dose administration and at $7^{\text {th }}$ and $14^{\text {th }}$ day of the study. On the $14^{\text {th }}$ day of the experimental procedure, all the animals in each group were anesthetized to collect the blood sample from the retro-orbital vein for the assessment of haematological parameters. The experimental animals were sacrificed by cervical dislocation for histology examination of various organs [14].

\section{Haematology and Biochemistry}

Haematology and biochemistry tests were performed by collecting blood from the retro orbital plexus by giving light anaesthesia to the mice then these blood samples were transferred into ethylenediaminetetraacetic acid (EDTA) coated vials for further analysis. Eurocount-TS Haematology Analyzer (Medsource Ozone Biomedicals; Haryana, India) was used for haematological examinations. Commercially available kits were employed for analyzing various parameters viz. haemoglobin, total leukocyte count (TLC), differential leukocyte counts (DLC), packed cell volume (PCV), mean corpuscular volume $(\mathrm{MCV})$, mean corpuscular hemoglobin $(\mathrm{MCH})$, mean corpuscular hemoglobin concentration (MCHC), platelet count, and red blood cell (RBC) count. Total bilirubin, total protein, albumin, serum glutamate oxaloacetate transaminase (SGOT), serum glutamate pyruvate transaminase (SGPT), and alkaline phosphatase (ALP) were measured for the liver function tests and blood urea, serum levels of creatinine, uric acid, potassium, sodium, calcium and phosphorous were measured for the kidney function tests. Analysis of body weight and feed intake represented physiological and feeding behaviour parameters [15].

\section{Histopathology}

On the $14^{\text {th }}$ day of the study, all animals in their respective groups were euthanized by cervical dislocation under anaesthesia. For Pathohistological diagnosis liver, kidney, heart, brain, intestine, pancreas, spleen and lungs were fixed in phosphate buffered formalin $(10 \%, \mathrm{v} / \mathrm{v})$ and the tissue sections were stained with haematoxylin and eosin (H\&E) for observation under light microscopy.

\section{Statistical Analysis}

For statistical analysis, the study results were evaluated by one-way analysis of variance (ANOVA). Values were expressed as mean \pm standard deviation (SD) and $\mathrm{p}<0.05$ was considered to be statistically significant.

\section{Results and Discussion}

Chitosan is an established safe, non-toxic, biocompatible and biodegradable polymer extensively used in pharmaceutical industry for diverse applications. Catechol conjugation of chitosan is a promising method for enhancing its mucoadhesive, sustained release and permeation enhancement properties. Catechol conjugation has been proposed to be an effective method for enhancing the mucoadhesive properties of chitosan. Synthesis procedure of chitosan-catechol conjugates was developed and the process parameters were optimized for the synthesis of conjugates. A SEM image of pure chitosan illustrates nonporous surface roughness with rigid flake like appearance (Figure 2A). However, a SEM image of chitosan-catechol conjugate shows the presence of interconnected fibrous structures (Figure 2B). Images obtained from a SEM were evaluated to study the surface morphology of the modified chitosan. Enhanced mucoadhesive potential of chitosan-catechol conjugates may be attributed to physical interaction of fibrous structure with mucin chains leading to strong physical interactions between mucin and polymer. 

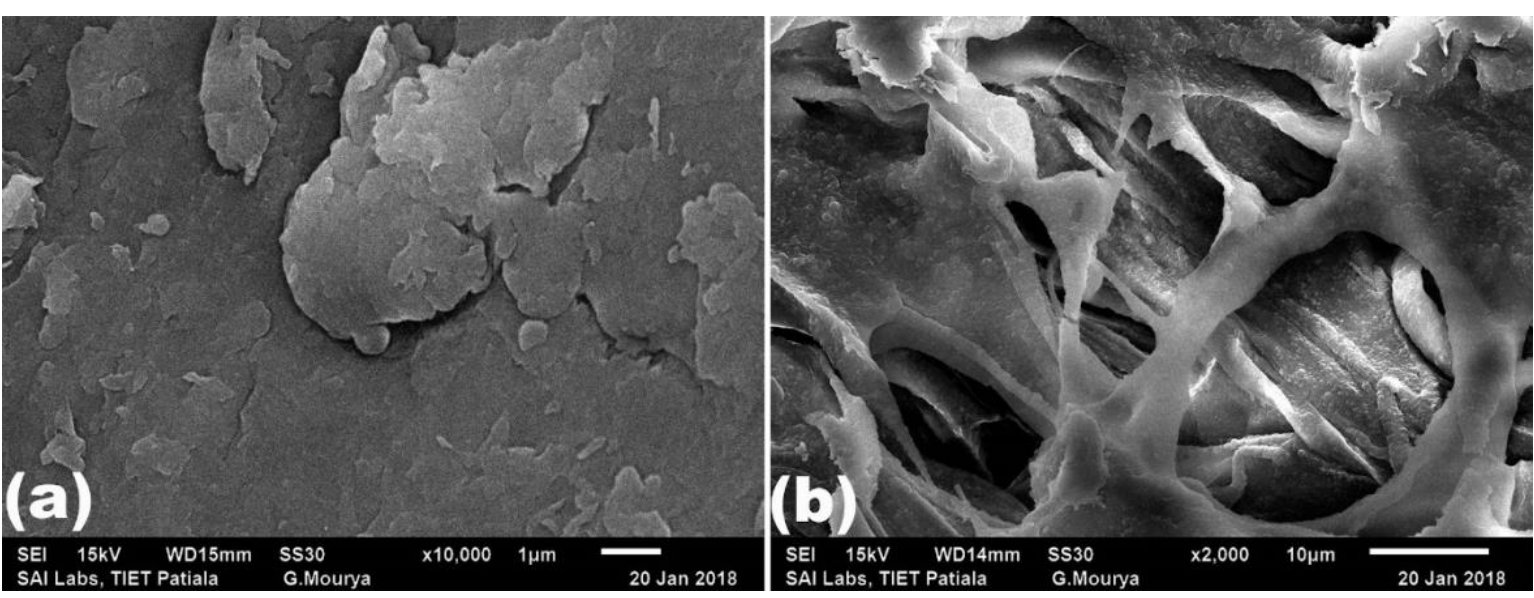

Figure 2. Scanning electron microscope (SEM) images of (A) chitosan and (B) chitosan-catechol conjugates.

The FT-IR spectra (Figure 3A) of chitosan shows characteristics peaks at 3,363 $\mathrm{cm}^{-1}$ attributed to $-\mathrm{OH}$ and $-\mathrm{NH}_{2} \mathrm{stretching}$, $1,655 \mathrm{~cm}^{-1}$ due to amide I band, $1,593 \mathrm{~cm}^{-1}$ due to amide II vibration and peaks between 1,000-1,200 $\mathrm{cm}^{-1}$ attributed to symmetric stretching of -C-O-C-. In the FT-IR spectra of chitosan-catechol conjugate (Figure 3B), shifting and relative increase in intensity of peak at $1,567 \mathrm{~cm}^{-1}$ indicates the successful conjugation between carboxylate group of hydrocaffeic acid and amine group of chitosan. In addition, appearance of sharp peaks at $2917 \mathrm{~cm}^{-1}$ and $1079 \mathrm{~cm}^{-1}$ further points toward successful coupling of chitosan with catechol moiety.
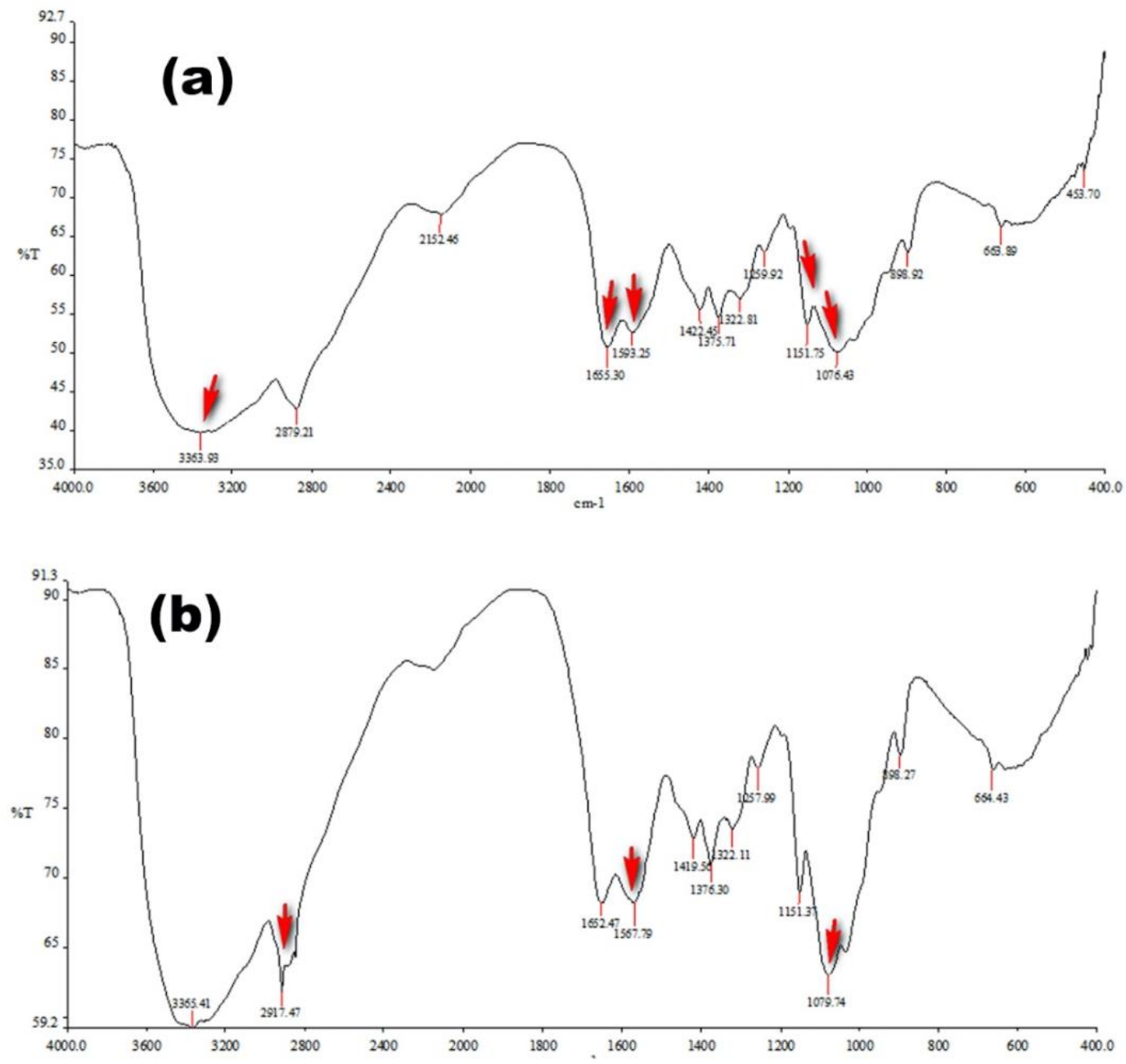

Figure 3. The Fourier-transform infrared (FT-IR) spectra of (A) chitosan and (B) chitosan-catechol conjugate. 
Pure chitosan indicates a XRD peak at $20.09{ }^{\circ} 2 \theta$ as shown in Figure 4A. Appearance of a XRD peak at $24.45{ }^{\circ} 2 \theta$ in chitosan-catechol conjugate indicates enhancement of amorphous property of the conjugate as shown in Figure $4 \mathrm{~B}$. This could be related to the enhanced mucoadhesive property of the conjugates. FT-IR and XRD studies clearly point out toward successful modification of chitosan by catechol modification.
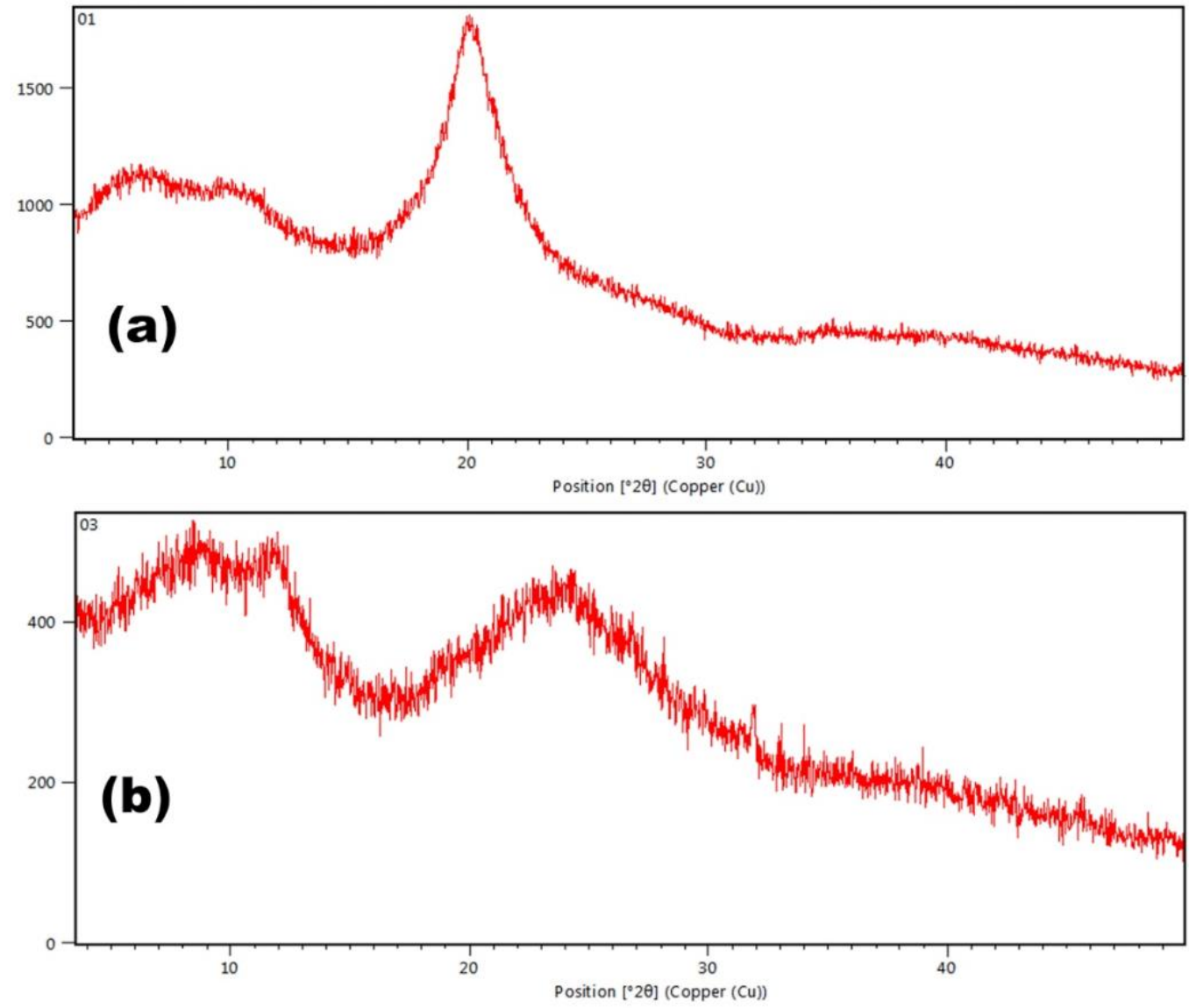

Figure 4. X-ray power diffraction (XRD) pattern of $(\mathrm{A})$ chitosan and $(\mathrm{B})$ chitosan-catechol conjugate.

\section{Haematology and Biochemistry}

Chitosan-catechol conjugates have been developed as a long lasting mucoadhesive polymer. No toxicity data was available for chitosan-catechol conjugates, so the present study was designed to evaluate single dose acute toxicity of chitosan-catechol conjugates in Swiss albino mice. It was observed that the modified polymer at oral dosages of 300 and $2,000 \mathrm{mg} / \mathrm{kg}$ body weight exhibited LD50 (lethal dose $50 \%$ ) value to be 2,000 $\mathrm{mg} / \mathrm{kg}$ body weight as per the study performed according to OECD 423 guidelines.

Results of haematological tests, kidney function tests, liver function tests and physiological and feeding behaviour parameters are shown in Table 2 . The findings suggested that there was no significant difference between the control and toxicity study groups (300 and 2,000 $\mathrm{mg} / \mathrm{kg}$ body-weight).

In this study, no significant changes were observed in low dose $(300 \mathrm{mg} / \mathrm{kg})$ and high dose $(2,000 \mathrm{mg} / \mathrm{kg}) \mathrm{of}$ synthesized chitosan-catechol conjugates.

Analysis of different clinical biochemistry parameters could be evaluated for correlating with acute toxicity potential of test substance. For toxicity evaluation, structural and functional stability of liver and kidney should be assessed. Liver is an important organ which plays diverse functions including production of different biochemical required for digestion, plasma protein synthesis, glycogen storage, hormone production, decomposition of red blood cells and detoxification. Elevated levels of liver specific enzymes, alanine aminotransferase (ALAT) and aspartate aminotransferase (ASAT) are direct indicators of hepatobiliary diseases and/or hepato-toxicity induced by drugs/chemicals/excipients.

As evident from the results in table 2, no changes were observed in all the treatment groups for liver and kidney function tests indicating that chitosan-catechol conjugates have no toxic effect on hepatic activity and the renal functioning. Similarly, the levels of serum electrolytes were found to be within range in all treatment groups when compared with the control group. 
Table 2. Single dose acute oral toxicity study exhibiting the effect of chitosan-catechol on haematological parameters, liver profile, kidney profile and behavioural test in experimental mice

\begin{tabular}{|c|c|c|c|}
\hline Parameters & Control & $\begin{array}{c}\text { Chitosan- } \\
\text { catechol } \\
(300 \mathrm{mg} / \mathrm{kg}) \\
\end{array}$ & $\begin{array}{c}\text { Chitosan- } \\
\text { catechol } \\
(2,000 \mathrm{mg} / \mathrm{kg}) \\
\end{array}$ \\
\hline \multicolumn{4}{|c|}{ Haematological test } \\
\hline $\mathrm{Hb}$ (Haemoglobin) (g/dL) & $11.5 \pm 0.5$ & $12.5 \pm 0.2$ & $12.2 \pm 0.4$ \\
\hline TLC (Total leukocyte count) (cell/cum) & $5,800 \pm 100.1$ & $5,780 \pm 106.7$ & $5,850 \pm 109.5$ \\
\hline \multicolumn{4}{|l|}{ DLC (Differential leukocyte count) (\%) } \\
\hline Neutrophil & $48.5 \pm 1.2$ & $47.8 \pm 1.8$ & $48.9 \pm 1.5$ \\
\hline Lymphocyte & $41.0 \pm 1.4$ & $42.5 \pm 1.0$ & $41.8 \pm 1.6$ \\
\hline Monocyte & $2.8 \pm 0.4$ & $3.0 \pm 0.5$ & $3.1 \pm 0.2$ \\
\hline Eosinophil & $1.9 \pm 0.2$ & $2.0 \pm 0.3$ & $1.8 \pm 0.4$ \\
\hline Basophil & $0.8 \pm 0.1$ & $1.0 \pm 0.2$ & $1.1 \pm 0.2$ \\
\hline PCV (Packed cell volume) (\%) & $42.8 \pm 1.5$ & $44.0 \pm 1.1$ & $44.4 \pm 1.3$ \\
\hline MCV (Mean corpuscular volume) (fL) & $81.8 \pm 2.4$ & $78.5 \pm 2.8$ & $78.9 \pm 2.5$ \\
\hline MCH (Mean corpuscular hemoglobin) (pg/cell) & $27.4 \pm 1.8$ & $26.1 \pm 0.9$ & $26.0 \pm 1.1$ \\
\hline $\begin{array}{l}\text { MCHC (mean corpuscular haemoglobin } \\
\text { concentration) }(\mathrm{g} / \mathrm{dL})\end{array}$ & $33.0 \pm 1.5$ & $33.2 \pm 2.0$ & $32.8 \pm 1.8$ \\
\hline Platelet count (million/ $\mathrm{cm}^{2}$ ) & $1.92 \pm 0.4$ & $1.95 \pm 0.2$ & $1.90 \pm 0.3$ \\
\hline RBC count (million/ $\left.\mathrm{cm}^{2}\right)$ & $4.6 \pm 0.6$ & $4.3 \pm 0.4$ & $4.5 \pm 0.7$ \\
\hline \multicolumn{4}{|c|}{ Liver function test } \\
\hline Total bilirubin (mg/dL) & $0.62 \pm 0.08$ & $0.64 \pm 0.05$ & $0.60 \pm 0.09$ \\
\hline Total protein $(\mathrm{g} / \mathrm{dL})$ & $6.6 \pm 0.2$ & $6.5 \pm 0.1$ & $6.7 \pm 0.2$ \\
\hline Albumin (g/dL) & $3.8 \pm 0.2$ & $4.0 \pm 0.3$ & $4.1 \pm 0.1$ \\
\hline $\begin{array}{l}\text { SGOT (serum glutamic-oxaloacetic } \\
\text { transaminase) (IU/L) }\end{array}$ & $80.4 \pm 1.4$ & $81.6 \pm 1.1$ & $81.2 \pm 1.5$ \\
\hline $\begin{array}{l}\text { SGOT (serum glutamic-pyruvic transaminase) } \\
\text { (IU/L) }\end{array}$ & $37.7 \pm 1.8$ & $39.0 \pm 1.7$ & $38.2 \pm 1.4$ \\
\hline ALP (alkaline phosphatase) (IU/L) & $129.5 \pm 4.0$ & $125.1 \pm 2.6$ & $126.4 \pm 3.9$ \\
\hline \multicolumn{4}{|c|}{ Kidney function test } \\
\hline Blood urea (mg/dL) & $34.2 \pm 2.5$ & $33.8 \pm 2.0$ & $33.9 \pm 1.5$ \\
\hline Serum creatinine $(\mathrm{mg} / \mathrm{dL})$ & $0.9 \pm 0.08$ & $1.0 \pm 0.06$ & $0.8 \pm 0.07$ \\
\hline Serum uric acid (mg/dL) & $4.8 \pm 0.4$ & $4.5 \pm 0.1$ & $4.7 \pm 0.8$ \\
\hline Serum potassium (mEq/dL) & $3.9 \pm 0.1$ & $4.0 \pm 0.3$ & $3.8 \pm 0.2$ \\
\hline Serum sodium $(\mathrm{mEq} / \mathrm{dL})$ & $142.2 \pm 2.7$ & $140.5 \pm 1.5$ & $146.1 \pm 3.2$ \\
\hline Serum calcium $(\mathrm{mEq} / \mathrm{dL})$ & $8.8 \pm 0.2$ & $8.6 \pm 0.7$ & $8.4 \pm 0.3$ \\
\hline Serum phosphorous (mEq/dL) & $3.8 \pm 0.1$ & $4.0 \pm 0.2$ & $4.1 \pm 0.1$ \\
\hline \multicolumn{4}{|c|}{ Physiological and feeding behaviour parameters } \\
\hline \multicolumn{4}{|l|}{ Body weight } \\
\hline $0^{\text {th }}$ day $(\mathrm{g})$ & $32.5 \pm 1.2$ & $30.7 \pm 1.4$ & $31.3 \pm 1.1$ \\
\hline $14^{\text {th }}$ day $(\mathrm{g})$ & $32.2 \pm 1.8$ & $30.0 \pm 1.0$ & $31.1 \pm 1.6$ \\
\hline \multicolumn{4}{|l|}{ Feed intake } \\
\hline $0^{\text {th }}$ day $(\mathrm{g} /$ day) & $1.1 \pm 0.5$ & $1.4 \pm 0.7$ & $1.2 \pm 0.3$ \\
\hline $14^{\text {th }}$ day (g/day) & $1.2 \pm 0.2$ & $1.3 \pm 0.4$ & $1.0 \pm 0.6$ \\
\hline
\end{tabular}

Kidneys are essential organs performing homeostatic activities such as maintenance of acid-base balance, regulation of electrolytes and maintaining the balance of salt and water for blood pressure. Kidneys act as a natural filter for the blood and excrete urine, ammonium compounds and urea, which are mainly responsible for the reabsorption of 
glucose, water and amino acids. For evaluating nephrotoxicity and renal functioning, assessment of parameters like serum creatinine, urine creatinine and urine microprotein are commonly used as the indicators.

\section{Histopathology Study}

No significant changes in the histopathological observations of brain, liver, heart, spleen, intestine, lungs, pancreas and kidneys of mice treated with two doses ( 300 and 2,000 mg/kg body-weight) of chitosan-catechol conjugates as shown in Figure 5.

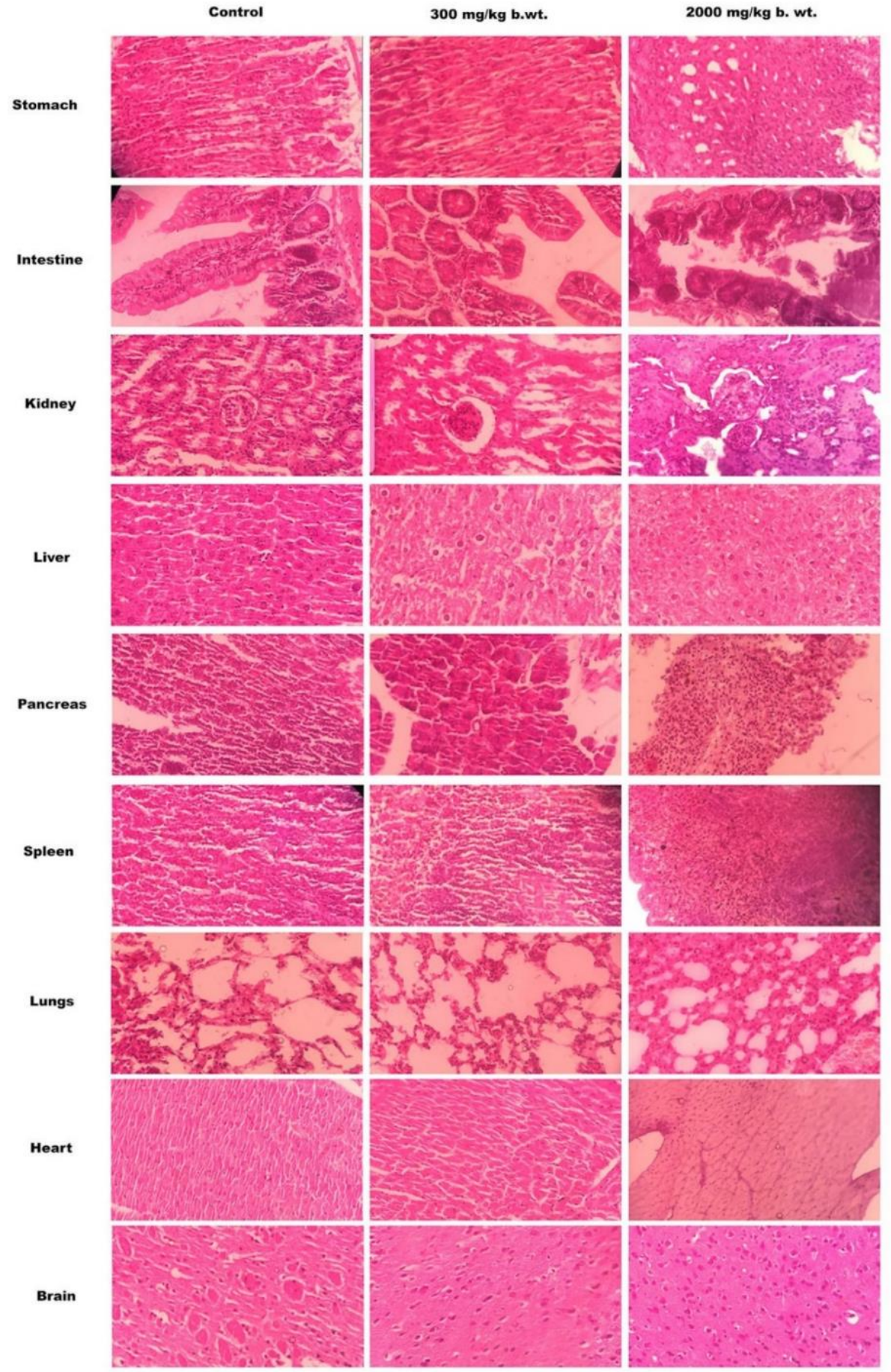

Figure 5. Photomicrographs of histological examination of transverse sections stomach, intestine, kidney, liver, pancreas, spleen, lungs, heart and brain tissues of mice. Column 1: control; column 2: $300 \mathrm{mg} / \mathrm{kg}$ body-weight of chitosan-catechol conjugates; column 3: 2,000 mg/kg body-weight of chitosan-catechol conjugates. 
Pathohistological analysis was performed for evaluating the structural stability of different body organs after oral administration of chitosan-catechol conjugates in different doses per unit body weight of the animal. As being evident in Figure 5, no ulcerative spots were noticed in the stomach after oral administration of the synthesized polymer. Histopathology of sections of intestine sections of treated mice showed similar normal architecture as that of control mice. The appearance of radiating hepatic cells in the liver sections of both control and polymer treated slides indicates no hepatic cell toxicity induced by the polymer.

Furthermore, the sections of the kidney tissue (Figure 5) showed the presence of Bowman's capsule surrounding the renal corpuscle and simple cuboidal epithelium lining the kidney tubules. A large cellular mass appears to be of the glomerulus and tuft of capillaries. These observations indicate no renal toxic effects of the modified polymer after peroral administration. No histological changes observed in the slides of spleen, heart, brain, pancreas and lungs of the treated animals when compared with the respective slides of control animals, indicating non-toxic ability of the chitosan-catechol conjugates in the selected dose.

\section{Conclusions}

It was concluded that chitosan-catechol conjugates which were given perorally at doses of 300 and 2,000 mg/kg body-weight in Swiss albino mice depicted no signs of toxicity based on observations related to general symptoms, body weight, death rate, food consumption, blood biochemistry, haematology, and histopathological findings. Therefore, on the basis of findings of acute oral toxicity, it was established that chitosan-catechol conjugates when administered perorally at single dose $(2,000 \mathrm{mg} / \mathrm{kg}$ body-weight) are nontoxic in Swiss albino mice.

\section{Acknowledgement}

Authors acknowledge institution facilities support extended by Chitkara College of Pharmacy, Chitkara University Punjab, India

\section{Conflict of interest}

The authors declare no conflict of interest regarding the publication of this manuscript.

\section{CRediT author statement}

LK: Conceptualization, Methodology, Writing-Original draft preparation, Editing; RR: Experimentation, Investigation, Visualization, Results compilation; AKT: Experimentation, Investigation, Visualization, Results compilation; IBS: Data curation and analysis, Supervision, Reviewing, Editing

\section{ORCID}

Loveleen Kaur: 0000-0002-7110-8399

Ajay Kumar Thakur: 0000-0002-2754-4759

Inderbir Singh: 0000-0002-1860-4246

\section{References}

[1] Baldrick P. Pharmaceutical excipient development - a preclinical challenge. In: Katdare A and Chaubal MV (eds.) Excipient development for pharmaceutical, biotechnology, and drug delivery systems. Informa Healthcare; 2006, 1536.

[2] Velagaleti R, DeMerlis CC, Brock W, Osterberg R, Goldring JM. Regulatory update: The IPEC novel excipient safety evaluation procedure. Pharma Technol 2009;33(11):1-6.

[3] Center for Drug Evaluation and Research (CDER). Guidance for industry: nonclinical studies for development of pharmaceutical excipients. 2005 [cited 2020 Mar 1]. Available from: www.fda.gov/cder/guidance

[4] Yeul VS, Rayalu SS. Unprecedented chitin and chitosan: a chemical overview. J Polym Environ 2013;21(2):606-614. DOI: 10.1007/s10924-012-0458-x

[5] Kumar MNVR. A review of chitin and chitosan applications. React Funct Polym 2000;46(1):1-27.

[6] Badhe RV, Nanda RK, Chejara DR, Choonara YE, Kumar P, du Toit LC, et al. Microwave-assisted facile synthesis of a new tri-block chitosan conjugate with improved mucoadhesion. Carbohydr Polym 2015;130(5):213-221. 
[7] Baldrick P. The safety of chitosan as a pharmaceutical excipient. Regulatory Toxicol Pharmacol 2010;56(3):290-299.

[8] Kim S-K, Park P-J, Yang H-P, Jan S-S. Subacute toxicity of chitosan oligosaccharide in sprague-dawley rats. ArzneimittelForschung 2001;51(9):769-774.

[9] Mukhopadhyay P, Bhattacharya S, Nandy A, Bhattacharyya A, Mishra R, Kumdu PP. Assessment of in vivo chronic toxicity of chitosan and its derivates used as oral insulin carrier. Toxicol Res 2015;4(2):281-290.

[10] Yang Z, Han B, Fu D, Liu W. Acute toxicity of high dosage carboxymethyl chitosan and its effect on the blood parameters in rats. J Mater Sci Mater Med 2012;23(2):457-462.

[11] Singh I, Rana V. Enhancement of mucoadhesive property of polymers for drug delivery applications: a critical review. Rev Adhesion Adhesives 2013;2:271-290.

[12] Kaur L, Singh I. Chitosan-catechol conjugates-A novel class of bioadhesive polymers: a critical review. Rev Adhesion Adhesives 2019;7(1):51-67.

[13] Kim KR, Kim K, Ryu JH, Lee H-S. Chitosan-catechol: A polymer with long lasting mucoadhesive properties. Biomaterials 2015;52:161-170.

[14] Schmitt D, Tran N, Riefler S, Jacoby J, Merkel D, Marone P et al. Toxicologic evaluation of modified gum acacia: Mutagenicity, acute and subchronic toxicity. Food Chem Toxicol 2008;46(3):1048-1054.

[15] Lee H, Park K. Acute toxicity of benzalkonium chloride in Balb/c mice following intratracheal instillation and oral administration. Environ Anal Health Toxicol 2019;34(3):e2019009. 